\title{
Factors associated with successful magnetic resonance-guided focused ultrasound treatment: efficiency of acoustic energy delivery through the skull
}

\author{
Won Seok Chang, MD, ${ }^{1}$ Hyun Ho Jung, MD, ${ }^{1}$ Eyal Zadicario, MSc, ${ }^{2}$ Itay Rachmilevitch, BSc, ${ }^{2}$ \\ Tal Tlusty, BSc, ${ }^{2}$ Shuki Vitek, PhD, ${ }^{2}$ and Jin Woo Chang, MD, PhD ${ }^{1}$ \\ ${ }^{1}$ Department of Neurosurgery, Brain Research Institute, Yonsei University College of Medicine, Seoul, Korea; and ${ }^{2}$ InSightec \\ Ltd., Tirat Carmel, Israel
}

\begin{abstract}
OBJECTIVE Magnetic resonance-guided focused ultrasound surgery (MRgFUS) was recently introduced as treatment for movement disorders such as essential tremor and advanced Parkinson's disease (PD). Although deep brain target lesions are successfully generated in most patients, the target area temperature fails to increase in some cases. The skull is one of the greatest barriers to ultrasonic energy transmission. The authors analyzed the skull-related factors that may have prevented an increase in target area temperatures in patients who underwent MRgFUS.

METHODS The authors retrospectively reviewed data from clinical trials that involved MRgFUS for essential tremor, idiopathic PD, and obsessive-compulsive disorder. Data from 25 patients were included. The relationships between the maximal temperature during treatment and other factors, including sex, age, skull area of the sonication field, number of elements used, skull volume of the sonication field, and skull density ratio (SDR), were determined.
\end{abstract}

RESULTS Among the various factors, skull volume and SDR exhibited relationships with the maximum temperature. Skull volume was negatively correlated with maximal temperature $\left(p=0.023, r^{2}=0.206, y=64.156-0.028 x\right.$, whereas SDR was positively correlated with maximal temperature $\left(p=0.009, r^{2}=0.263, y=49.643+11.832 x\right)$. The other factors correlate with the maximal temperature, although some factors showed a tendency to correlate.

CONCLUSIONS Some skull-related factors correlated with the maximal target area temperature. Although the number of patients in the present study was relatively small, the results offer information that could guide the selection of MRgFUS candidates.

http://thejns.org/doi/abs/10.3171/2015.3.JNS142592

KEY WORDS focused ultrasound; magnetic resonance; skull; treatment; functional neurosurgery

$\mathrm{S}$ INCE the time that Spiegel and colleagues introduced stereotactic devices for the treatment of humans, there have been many advances in stereotactic procedures for treating neurological diseases, including Parkinson's disease (PD), essential tremor (ET), chronic neuropathic pain, and obsessive-compulsive disorder (OCD) ${ }^{21}$ Radiofrequency (RF) thermal ablation can create lesions in deep brain structures more easily than earlier modalities such as chemical ablation using alcohol. RF was widely applied to treat movement and psychiatric disorders until deep brain stimulation surgery was introduced. ${ }^{8,11,15}$ However, RF lesioning procedures were found to be associated with a relatively high rate of permanent complications, especially when performed in both hemispheres. ${ }^{19}$ As an alternative to RF thermal lesioning, bilateral deep brain stimulation has been shown to have a high success rate and an acceptable risk of complications. ${ }^{23}$

Although the risks associated with current neurosurgical procedures for movement and psychiatric disorders are considered acceptable, there are many potential procedure-, hardware-, and anesthesia-related complications that must be considered by both physicians and patients. ${ }^{7}$ To overcome these shortcomings, a less invasive technique using ultrasonic energy was developed. Magnetic reso-

ABBREVIATIONS Emax = maximal energy required to achieve Tmax; ET = essential tremor; MRgFUS = magnetic resonance-guided focused ultrasound surgery; OCD = obsessive-compulsive disorder; $\mathrm{PD}=$ Parkinson's disease; $\mathrm{RF}$ = radiofrequency; $\mathrm{SDR}$ = skull density ratio; $T$ max = maximum temperature.

SUBMITTED November 12, 2014. ACCEPTED March 12, 2015.

INCLUDE WHEN CITING Published online September 11, 2015; DOI: 10.3171/2015.3.JNS142592. 
nance-guided focused ultrasound surgery (MRgFUS) is currently being tested in human clinical trials to determine its efficacy and safety for treating ET, PD, chronic neuropathic pain, and OCD. $1,3,5,13,14$ The currently available results suggest that MRgFUS achieves a high success rate with a very low rate of permanent complications. However, when temperature in the target area fails to increase in some patients, lesioning is not achieved. This failure occurs in only a small proportion of patients who undergo MRgFUS, and there is no available information regarding potential reasons. In this study, we analyzed various patient- and treatment-related factors during MRgFUS and tried to identify the factors associated with poor treatment outcomes.

\section{Methods}

This study was conducted under the permission and supervision of the Severance Hospital institutional review board (Seoul, Korea). We retrospectively reviewed data from patients who underwent MRgFUS at our institute. For MRgFUS of ET, we choose the ventralis intermedius nucleus of the thalamus as a target region. ${ }^{3}$ The bilateral anterior capsules were targeted for treatment of OCD, and the posteroventral aspect of the globus pallidus interna was lesioned to treat idiopathic PD. During the procedures, we elevated the sonication energy in a stepwise fashion until patients experienced sufficient symptom improvement or until the temperature did not increase even with higher sonication energy. The inclusion criteria for this study were patients who completed MRgFUS in which there was a maximum temperature (Tmax) at the target area of $50^{\circ} \mathrm{C}$ or greater on MR thermometry and clearly visible lesions on postoperative MRI. Data from 25 patients (15 with ET, 1 with idiopathic PD, and 9 with OCD) were used for this study.

We collected patient-specific and treatment-related data. The patient factors included sex, age, skull volume in treatment area, and skull density ratio (SDR). The SDR was defined as the ratio between the mean values in Hounsfield units for marrow and cortical bone, which was calculated as follows: 1) The planned locations of the target and the transducer were marked on CT scans, and the surface of the skull was subdivided into small sections. 2) For each skull subsection, multiple parallel rays were created and the CT values along each ray were used to create local density profiles. 3) For each profile, the trabecular $\mathrm{CT}$ value (local minimum CT value in the profile) and the cortex CT value (average of inner and outer cortices) were computed. 4) The "local" SDR was the average of the ratios between the trabecular and cortex CT values of all of the profile samples.

Treatment-related parameters included skull area in the treatment field, average skull thickness, total number of elements actually used during treatment, and number of sonication elements that penetrated the skull by an incidence angle larger than $25^{\circ}$. Both patient- and treatmentrelated factors were compared with the target area Tmax measured by MR thermometry.

For statistical analysis, nonparametric Mann-Whitney U-tests were used to compare the mean values accord- ing to patient sex. Correlations between other factors and Tmax were calculated by linear regression methods using SPSS v.20 (IBM Corp.).

All clinical trials with MRgFUS treatments were performed with the approval of the Yonsei University College of Medicine and the Korean Food and Drug Administration.

\section{Results}

Initially, we attempted to treat 28 patients with MRgFUS. However, 3 patients with ET failed to show a sufficient temperature increase $\left(<50^{\circ} \mathrm{C}\right)$. Although this failure may have resulted from patient- or treatmentrelated factors, other factors including technical failure or device malfunction may have also played a role because these patients underwent MRgFUS in the early stage of the study period. Therefore, data from these patients were excluded from this study to facilitate analysis, as a temperature increase over $50^{\circ} \mathrm{C}$ guaranteed that ultrasonic energy was successfully transmitted into the skull, even though Tmax varied among patients.

The mean Tmax (range) was $55.4^{\circ} \mathrm{C}\left(52^{\circ} \mathrm{C}-62^{\circ} \mathrm{C}\right)$. The mean Tmax values for MRgFUS thalamotomy and anterior capsulotomy were $55.1^{\circ} \mathrm{C}\left(53^{\circ} \mathrm{C}-62^{\circ} \mathrm{C}\right)$ and $55.4^{\circ} \mathrm{C}$ $\left(52^{\circ} \mathrm{C}-61^{\circ} \mathrm{C}\right)$, respectively. There was no statistical difference in Tmax between the two procedure types (MannWhitney U-test, $\mathrm{p}=0.907$; Table 1).

The analysis of Tmax and patient-related factors indicated that skull volume in the treatment area and SDR were closely correlated with Tmax. Skull volume and Tmax were negatively correlated $\left(\mathrm{p}=0.023, \mathrm{r}^{2}=0.206\right.$, $\mathrm{y}=64.156-0.028 \mathrm{x})$, and SDR and Tmax were positively correlated $\left(p=0.009, r^{2}=0.263, y=49.643+11.832 x\right)$. Other factors such as age and sex were not associated with Tmax (Fig. 1). However, skull volume showed correlation with mean skull thickness, which implies that mean skull thickness could be useful for predicting the degree of Tmax, if more data were available (Table 2).

None of the treatment-related factors were significantly related to Tmax during MRgFUS, although the number of elements during sonication tended to correlate with Tmax without exhibiting statistical significance $\left(\mathrm{p}=0.065, \mathrm{r}^{2}=\right.$ $0.141, \mathrm{y}=39.112+0.018 \mathrm{x})($ Table 3$)$.

We also analyzed the relationship between maximal energy required to achieve Tmax (Emax) and SDR-skull volume (Figs. 2 and 3). SDR and skull volume appeared to have a positive and negative correlation with Emax, respectively. Although there was no clear evidence of SDR or skull volume as a determinant for energy requirement to achieve sufficient Tmax, lower sonication energy appeared to be required for SDR $>0.45$ and skull volume $<320 \mathrm{~cm}^{3}$.

\section{Discussion}

In this study, we tried to identify the factors affecting temperature increase during MRgFUS, and our results indicate that skull volume and SDR in the treatment field seem to be closely related to temperature increase. During thermal lesioning, the degree of temperature increase is crucial for successful treatment. Generally the extent of thermal lesioning is determined by two factors: the degree 
TABLE 2. Correlation between SDR, skull volume, and each patients-related factor*

\begin{tabular}{lccccc}
\hline \multicolumn{7}{c}{$\begin{array}{c}\text { Average } \\
\text { Skull } \\
\text { Factor }\end{array}$} & Skull Vol & Thickness & Skull Area & Age & SDR \\
\hline SDR & $p=0.144$ & $p=0.534$ & $p=0.310$ & $p=0.265$ & NA \\
\hline Skull vol & NA & $p=0.003 \dagger$ & $p=0.028 \dagger$ & $p=0.140$ & $p=0.144$ \\
\hline NA $=$ not applicable. & & & \\
$*$ & Boldface indicates a significant correlation $(p<0.05)$. & \\
$\dagger p<0.05$, multiple linear regression.
\end{tabular}

of Tmax and the duration of Tmax. For example, $1 \mathrm{sec}-$ ond at $57^{\circ} \mathrm{C}$ is known to be sufficient for tissue necrosis, whereas for tissue necrosis with a Tmax of $54^{\circ} \mathrm{C}, 3 \mathrm{sec}-$ onds is needed. ${ }^{18}$ Similar to other modalities for thermal lesioning, MRgFUS also could modulate the duration of Tmax by increasing the duration of sonication. However, the degree of Tmax showed limitations even with higher sonication energy. Because this phenomenon can directly affect the treatment results, identifying the factors related to temperature increase is crucial for selecting patients and improving treatment outcomes. In this study, it appeared that patient-related factors, particularly SDR and skull volume, more than treatment-related factors appeared to have been more correlated to energy transmission across the skull.

\section{Development of Treatment Technologies for Stereotactic and Functional Neurosurgery}

There have been various advances in procedures and technologies since neurosurgical interventions were first applied to treat CNS diseases. In the early 1990s, neurosurgical interventions for functional brain diseases were performed by open craniotomy and resection or disconnection of cortical areas. ${ }^{2,9,10}$ Although the efficacy of this techniques was acceptable, the associated mortality and morbidity rates were high, which limited the wide use of these procedures. Once Spiegel and colleagues invented the human stereotactic frame, it became possible to perform deep brain neurosurgical procedures with high accuracy and precision. This technical innovation changed neurosurgical procedures for functional brain diseases such as movement disorders, epilepsy, and psychiatric ill-

TABLE 3. Correlation between each factor and Tmax

\begin{tabular}{|c|c|}
\hline Factor & p Value* \\
\hline Skull vol $\left(\mathrm{cm}^{3}\right)$ & $p=0.023\left(r^{2}=0.206, y=64.156-0.028 x\right)$ \\
\hline Skull density ratio & $p=0.009\left(r^{2}=0.263, y=49.643+11.832 x\right)$ \\
\hline $\begin{array}{l}\text { No. of elements of } \\
\text { angle }>25^{\circ}\end{array}$ & $p=0.798\left(r^{2}=0.003, y=55.195+0.002 x\right)$ \\
\hline Skull area $\left(\mathrm{cm}^{2}\right)$ & $p=0.164\left(r^{2}=0.086, y=65.461-0.029 x\right)$ \\
\hline $\begin{array}{l}\text { Used elements for } \\
\text { Tmax }\end{array}$ & $p=0.065\left(r^{2}=0.141, y=39.112+0.018 x\right)$ \\
\hline $\begin{array}{l}\text { Mean skull thickness } \\
(\mathrm{mm})\end{array}$ & $p=0.567\left(r^{2}=0.024, y=57.606-0.397 x\right)$ \\
\hline Age & $p=0.838\left(r^{2}=0.002, y=55.022+0.006 x\right)$ \\
\hline
\end{tabular}

* Boldface indicates a significant correlation $(p<0.05)$. 

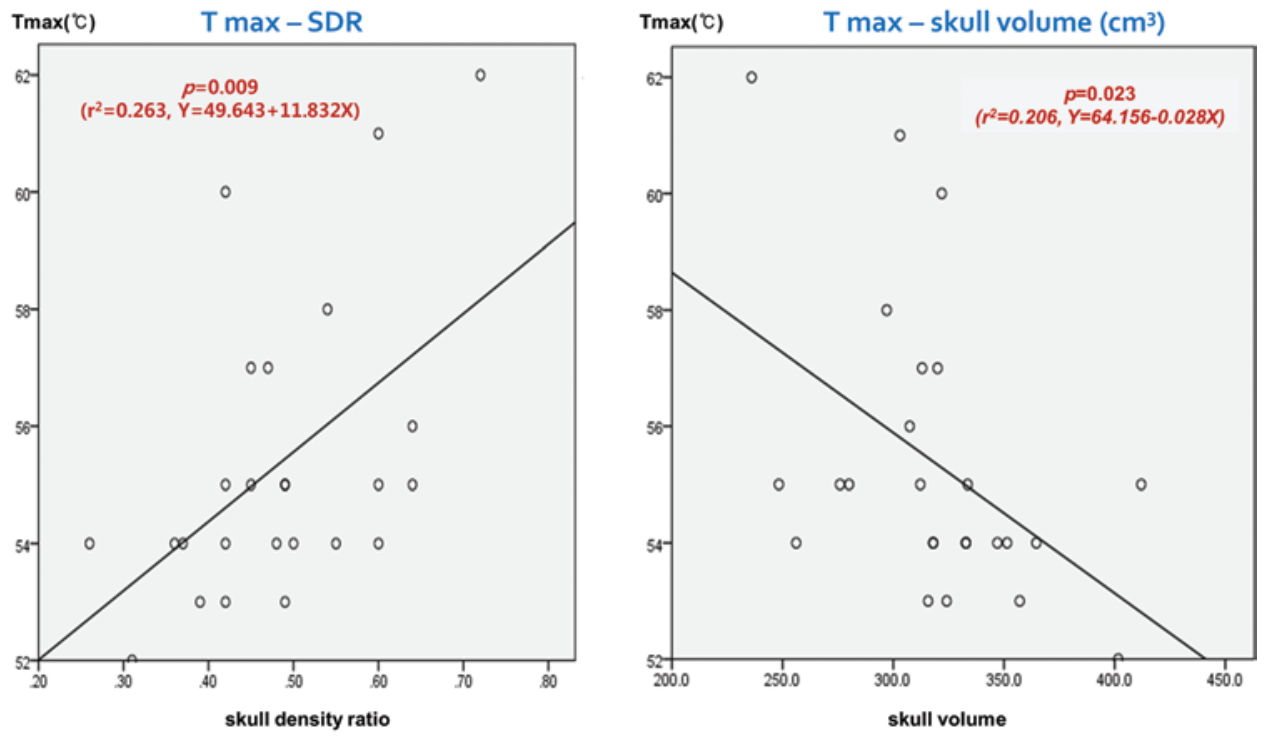

FIG. 1. Linear regression of skull volume, skull density ratio, and Tmax. Figure is available in color online only.

ness, including attempts at generating focal lesions in target areas.

Different modalities have been used for lesioning. RF ablation is widely performed because it can generate precise lesions with high reliability, but postoperative hemorrhages in deep brain nuclei or cortical areas have been reported in up to $35 \%$ of patients who undergo RF lesioning. ${ }^{22}$ Because infection and anesthesia-related complications also contribute to the surgical risk, the overall complication rate after RF lesioning should be considered, and safer modalities have been emphasized, especially for surgeries in patients with functional disorders. Gamma Knife radiosurgery was developed as a minimally invasive technique capable of precisely generating a focal lesion in the brain, and early candidates for its application were func-

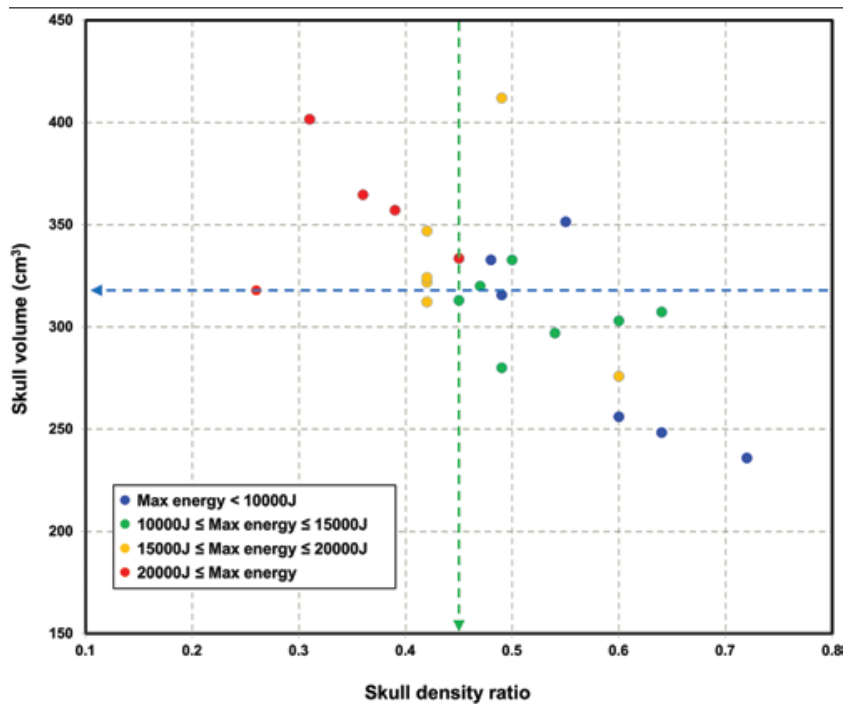

FIG. 2. Maximal energy requirement (J [joule]) for Tmax as function of SDR and skull volume (blue dotted line indicates a skull volume of 320 $\mathrm{cm}^{3}$ and green dotted line an SDR of 0.45$)$. Figure is available in color online only. tional disorders including cancer pain and some psychiatric illnesses. ${ }^{6,12}$ Although Gamma Knife radiosurgery, especially in Gamma Knife thalamotomy, have achieved an efficacy similar to RF lesioning for movement disorders, there are important issues to consider, including the time interval between treatment and effect, variability of the target area reaction, and inability to predict side effects for specific patients., ${ }^{4,17}$ Furthermore, the complication rate associated with Gamma Knife radiosurgery in patients with movement disorders has been reported to be as high as $50 \%$, although the reported complications were not fatal.
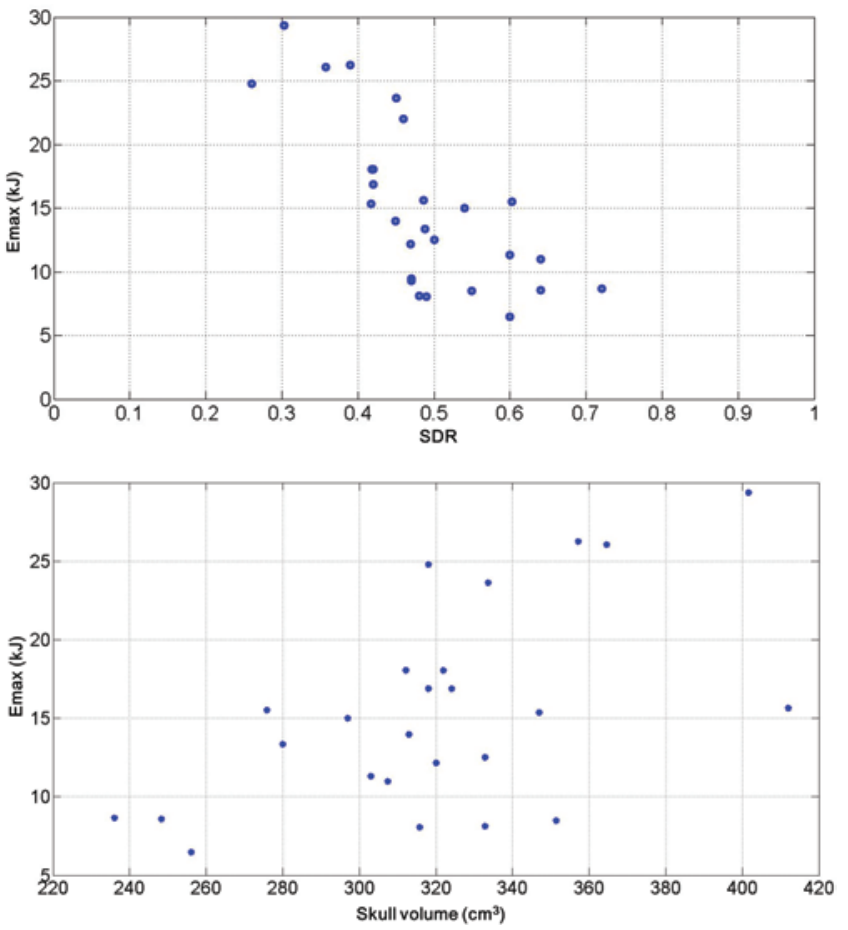

FIG. 3. Relationship between Emax according to SDR (upper) and skull volume (lower). Figure is available in color online only. 


\section{Recent Clinical Trials With MRgFUS: Potential and Limitations}

Progressive innovation in radiographic modalities and technical developments has enabled ultrasonic energy to be focused through the skull, leading to the application of MRgFUS for functional neurosurgery. Several clinical trials have examined the use of MRgFUS for thalamotomy. ${ }^{3,16}$ Reporting on the 1-year follow-up results for 15 patients who underwent MRgFUS thalamotomy, Elias et al. indicated that there were no hemorrhagic complications and only 1 serious adverse event, dysesthesia affecting a patient's index finger. ${ }^{5}$ Similarly, we previously reported that minimally invasive MRgFUS thalamotomy was effective and safe, but in some of our patients, the MRgFUS-induced thermal increase was not sufficient to create a lesion. ${ }^{3}$ Although an appropriate temperature increase was easily achieved with relatively low ultrasonic energy in some patients, others required higher ultrasonic energy to elevate the target area temperature.

\section{Factors Related to Thermal Rise: SDR and Skull Volume}

Scarce information regarding thermal increase was available during the clinical trial, and we assumed that skull variability might be related to the differences in thermal increase. We therefore measured the skull volume in the treatment area and found that temperature increase could be affected by skull volume. In the present study, we collected more data from other MRgFUS trials, which confirmed that skull volume in the treatment area was closely related to the thermal increase during MRgFUS surgery $\left(p=0.023, r^{2}=0.206, y=64.156-0.028 x\right)$. Although further investigation is needed to define skull volume cutoff values, this result suggests that measurement of skull volume should be an essential step when selecting candidates for MRgFUS.

Another factor found to limit energy transfer throughout the skull was SDR. As mentioned above, SDR is defined as the mean value for the ratio of Hounsfield units of marrow and cortical bone in the treatment area, with a lower SDR indicating that the Hounsfield unit value for cortical bone is much higher than that for bone marrow. When ultrasonic energy impacts different media, 3 different patterns of action are observed: absorption, reflection, and attenuation. ${ }^{20}$ All 3 effects can interrupt focused ultrasonic energy. It could thus be postulated that a larger skull volume results in a less temperature increase because bone absorbs ultrasonic energy at a rate 100 times higher than that of soft tissue. Reflection between different tissues also can reduce ultrasonic energy, and energy reflection is correlated with different acoustic impedances for various materials and the incidence angle of ultrasonic energy. Considering that acoustic impedance is calculated by multiplying the tissue density by the conduction velocity in tissue, the density difference within the skull can also affect energy reflection and contribute to the reduced temperature increase in patients with lower SDR $\left(\mathrm{p}=0.009, \mathrm{r}^{2}\right.$ $=0.263, y=49.643+11.832 x)($ Fig. 4). We did not observe a correlation between the incidence angle and temperature increase $(p=0.798)$, which could indicate that the incidence angle during MRgFUS surgery was not sufficient to cause reflection and affect focusing energy; however, further investigation is needed to test this hypothesis.

\section{Energy Requirement for Tmax According to SDR and Skull Volume}

Emax also should be considered before and during MRgFUS treatment because higher energy requirements could be problematic. There are 2 main parameters for modulating Emax during MRgFUS treatment: sonication power and sonication duration. Although tissue responses to increasing sonication power and duration may show some differences, increase in sonication power and duration could cause overheating of the skin/skull and prolonged treatment time may result in incomplete lesioning because of treatment intolerance of the patients. According to our results, both SDR and skull volume appeared to be related to Emax (Figs. 2 and 3). Because of insufficient sample size, we could not determine the cutoff values for SDR and skull volume recommendations for minimizing Emax. Nevertheless, it could be postulated that skull vol-

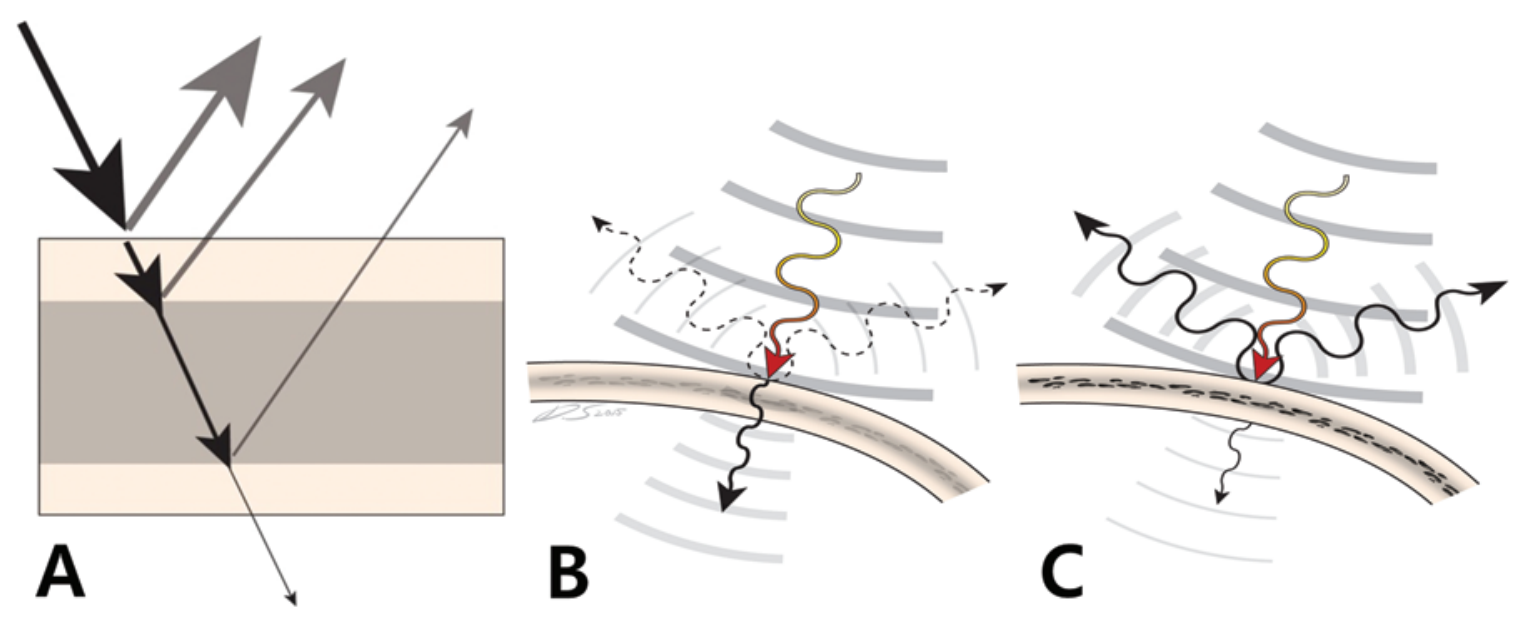

FIG. 4. Ultrasonic energy transmission across skull. A: Ultrasonic energy attenuation and reflection at cortical and marrow bone. B: Higher energy transmission across skulls with higher SDR. C: Lower energy transmission across skulls with lower SDR. Copyright Jin Woo Chang. Published with permission. Figure is available in color online only. 
ume less than $330 \mathrm{~cm}^{3}$ and/or SDR higher than 0.45 may predict a lower energy requirement during MRgFUS.

\section{Conclusions}

MRgFUS was introduced as a minimally invasive functional neurosurgical option, and recent trials have suggested acceptable clinical outcomes. However, the temperature increase associated with target area lesioning varies among patients. In the current study, we tried to identify the factors affecting temperature increase during MRgFUS. Our results indicate that skull volume and SDR in the treatment field seem to be closely related to temperature increase. This information would be expected to be helpful for the selection of patients for MRgFUS surgery and also for the optimization of the treatment outcomes.

\section{Acknowledgments}

This work was supported by the Yonsei University FutureLeading Research Initiative of 2014 (2014-22-0150). We are grateful to Dong-Su Jang, Medical Illustrator of the Medical Research Support Section, Yonsei University College of Medicine, for assistance in preparing the figures.

\section{References}

1. Bauer R, Martin E, Haegele-Link S, Kaegi G, von Specht M, Werner B: Noninvasive functional neurosurgery using transcranial MR imaging-guided focused ultrasound. Parkinsonism Relat Disord 20 (Suppl 1):S197-S199, 2014

2. Bucy P, Buchanan D: Athetosis. Brain 55:479-492, 1932

3. Chang WS, Jung HH, Kweon EJ, Zadicario E, Rachmilevitch I, Chang JW: Unilateral magnetic resonance guided focused ultrasound thalamotomy for essential tremor: practices and clinicoradiological outcomes. J Neurol Neurosurg Psychiatry 86:257-264, 2015

4. Elaimy AL, Arthurs BJ, Lamoreaux WT, Demakas JJ, Mackay AR, Fairbanks RK, et al: Gamma knife radiosurgery for movement disorders: a concise review of the literature. World J Surg Oncol 8:61, 2010

5. Elias WJ, Huss D, Voss T, Loomba J, Khaled M, Zadicario E, et al: A pilot study of focused ultrasound thalamotomy for essential tremor. N Engl J Med 369:640-648, 2013

6. Frighetto L, Bizzi J, D’Agostini Annes R, dos Santos Silva R, Oppitz P: Stereotactic radiosurgery for movement disorders. Surg Neurol Int 3 (Suppl 1):S10-S16, 2012

7. Hariz MI: Complications of deep brain stimulation surgery. Mov Disord 17 (Suppl 3):S162-S166, 2002

8. Hassenbusch SJ, Pillay PK, Barnett GH: Radiofrequency cingulotomy for intractable cancer pain using stereotaxis guided by magnetic resonance imaging. Neurosurgery 27:220-223, 1990

9. Horsley V: The function of the so-called motor area of the brain. BMJ 2:121-132, 1909

10. Horsley V: The surgery of the central nervous system. BMJ 2:1286-1292, 1890

11. Kim CH, Chang JW, Koo MS, Kim JW, Suh HS, Park IH, et al: Anterior cingulotomy for refractory obsessive-compulsive disorder. Acta Psychiatr Scand 107:283-290, 2003
12. Leksell L: Stereotactic radiosurgery. J Neurol Neurosurg Psychiatry 46:797-803, 1983

13. Lipsman N, Mainprize TG, Schwartz ML, Hynynen K, Lozano AM: Intracranial applications of magnetic resonanceguided focused ultrasound. Neurotherapeutics 11:593-605, 2014

14. Lipsman N, Schwartz ML, Huang Y, Lee L, Sankar T, Chapman M, et al: MR-guided focused ultrasound thalamotomy for essential tremor: a proof-of-concept study. Lancet Neurol 12:462-468, 2013

15. Mark VH, Ervin FR, Yakolev PI: Stereotactic thalamotomy. III. The verification of anatomical lesion sites in the human thalamus. Arch Neurol 8:528-538, 1963

16. Medel R, Monteith SJ, Elias WJ, Eames M, Snell J, Sheehan JP, et al: Magnetic resonance-guided focused ultrasound surgery: Part 2: A review of current and future applications. Neurosurgery 71:755-763, 2012

17. Niranjan A, Jawahar A, Kondziolka D, Lunsford LD: A comparison of surgical approaches for the management of tremor: radiofrequency thalamotomy, gamma knife thalamotomy and thalamic stimulation. Stereotact Funct Neurosurg 72:178184,1999

18. Sapareto SA, Dewey WC: Thermal dose determination in cancer therapy. Int J Radiat Oncol Biol Phys 10:787-800, 1984

19. Schuurman PR, Bosch DA, Bossuyt PM, Bonsel GJ, van Someren EJ, de Bie RM, et al: A comparison of continuous thalamic stimulation and thalamotomy for suppression of severe tremor. N Engl J Med 342:461-468, 2000

20. Shriki J: Ultrasound physics. Crit Care Clin 30:1-24, v, 2014

21. Spiegel EA, Wycis HT, Marks M, Lee AJ: Stereotactic apparatus for operations on the human brain. Science 106:349350,1947

22. Terao T, Takahashi H, Yokochi F, Taniguchi M, Okiyama R, Hamada I: Hemorrhagic complication of stereotactic surgery in patients with movement disorders. J Neurosurg 98:12411246, 2003

23. Weaver FM, Follett K, Stern M, Hur K, Harris C, Marks WJ $\mathrm{Jr}$, et al: Bilateral deep brain stimulation vs best medical therapy for patients with advanced Parkinson disease: a randomized controlled trial. JAMA 301:63-73, 2009

\section{Disclosure}

Mr. Zadicario, Mr. Rachmilevitch, Mr. Tlusty, and Dr. Vitek are employees of InSightec.

\section{Author Contributions}

Conception and design: JW Chang. Acquisition of data: WS Chang, Jung. Analysis and interpretation of data: WS Chang, Zadicario, Rachmilevitch. Drafting the article: WS Chang. Critically revising the article: JW Chang. Reviewed submitted version of manuscript: JW Chang, Jung. Administrative/technical/material support: Tlusty, Vitek. Study supervision: JW Chang.

\section{Correspondence}

Jin Woo Chang, Department of Neurosurgery, Brain Research Institute, Yonsei University College of Medicine, 205 Seongsanno, Seodaemun-gu, Seoul 120-752, Republic of Korea. email: jchang@yuhs.ac. 